\title{
Prevalence, screening and treatment of latent tuberculosis among oral corticosteroid recipients
}

\author{
To the Editor:
}

Tuberculosis guidelines identify individuals receiving the corticosteroid drug prednisone (or its equivalent) at a dose of $>15 \mathrm{mg} \cdot \mathrm{day}^{-1}$ for 2-4 weeks or more as a group at risk of tuberculosis if infected with Mycobacterium tuberculosis [1,2]. There is an eight-fold increased risk of developing active tuberculosis with such drugs at this dose [3]. However, there is no information on the epidemiology of latent tuberculosis infection (LTBI), screening and treatment among oral corticosteroid users. Tuberculosis guidelines recommend using a threshold of $\geqslant 5 \mathrm{~mm}$ induration to identify latent infection among oral corticosteroid recipients $[1,2]$ but this recommendation is not evidence-based. The purpose of this study was to describe the prevalence, screening and treatment of LTBI among oral corticosteroid recipients in the USA.

This was a cross-sectional study using US nationally representative, population-level data from the 19992000 National Health and Nutrition Examination Survey (NHANES). A description of the survey design and methodology appears elsewhere [4]. Self-reported medication receipt within the past month that required a prescription was collected by NHANES. Medication receipt was confirmed in $83.3 \%$ of participants through examiner inspection of prescription containers [5]. Survey participants who received any corticosteroid in an oral formulation within the past month were considered "recipients". Survey participants who did not receive any oral corticosteroids within the past month were considered "nonrecipients". Topical, inhaled or intra-articular corticosteroids were not included in this study. Information on total duration of corticosteroid receipt was collected but not dose. A single-step tuberculin skin test (TST) was implanted and available in 7317 participants (no age restriction), excluding individuals with a self-reported history of previous severe reaction to TST, active severe skin conditions over the arms, and a known or uncertain history of self-reported, doctor-diagnosed, active tuberculosis. NHANES also collected information on self-reported history of previous TST and receipt of antibiotic therapy for LTBI among individuals with a previously positive TST. The distribution of size of TST induration, and the prevalence of LTBI, previous TST screening and previous receipt of chemoprophylaxis for LTBI were described among corticosteroid recipients and nonrecipients.

Of the 7317 participants, $93(1.3 \%)$ were oral corticosteroid recipients and 7224 (98.7\%) were nonrecipients. The average duration of corticosteroid receipt was 1002 days, with $73 \%$ of recipients taking a corticosteroid for $\geqslant 2$ weeks. Significantly greater proportions of corticosteroid recipients versus nonrecipients were older in age, had a bacille Calmette-Guérin (BCG) vaccination scar, were obese, or had asthma, COPD or arthritis (data not shown). The distribution of the size of TST induration among corticosteroid recipients was leftward-shifted relative to nonrecipients, such that $87.5 \%$ of recipients had a TST induration of $0 \mathrm{~mm}$ versus $80.5 \%$ of nonrecipients (fig. 1). Compared with nonrecipients, there were nonsignificant trends towards fewer recipients demonstrating a positive TST, as defined by an induration $\geqslant 5 \mathrm{~mm}(3.1 \%$ versus $6.1 \%, \mathrm{p}=0.22)$. Assuming corticosteroid recipients have, at minimum, the same prevalence of LTBI as nonrecipients (i.e. $4.1 \%$ based on a TST cut-off of $\geqslant 10 \mathrm{~mm}$ ), a TST cut-off of $\sim 3.5 \mathrm{~mm}$ induration would be needed among recipients to denote positivity in order to obtain the same prevalence of LTBI. Compared with nonrecipients, a nonsignificant trend towards fewer recipients having undergone previous TST screening was found $(62.9 \%$ versus $71.6 \%, \mathrm{p}=0.07)$. Compared with nonrecipients, no corticosteroid recipients had received antibiotic prophylaxis $(0 \%$ versus $44.3 \%, \mathrm{p}=0.54)$.

The lower prevalence of LTBI among corticosteroid recipients versus nonrecipients is probably not real and, instead, a reflection of the drugs' known ability to suppress TST reactivity [6-9]. Given the evidence for TST suppression by systemic corticosteroids, TB guidelines recommend using a lower-than-usual TST threshold of $\geqslant 5 \mathrm{~mm}$ induration to denote positivity among corticosteroid recipients $[1,2]$ but this cut-off is not evidence-based. Our data demonstrate a TST cut-off of $\sim 3.5 \mathrm{~mm}$ induration would be needed among corticosteroid recipients to obtain the same prevalence of LTBI as in the general population. The findings of trends towards less TST screening and less chemoprophylaxis for positive TST cases among corticosteroid recipients are concerning, as these factors may be contributing to the development of active tuberculosis in this already tuberculosis-vulnerable population. Our finding of higher frequency of previous BCG 


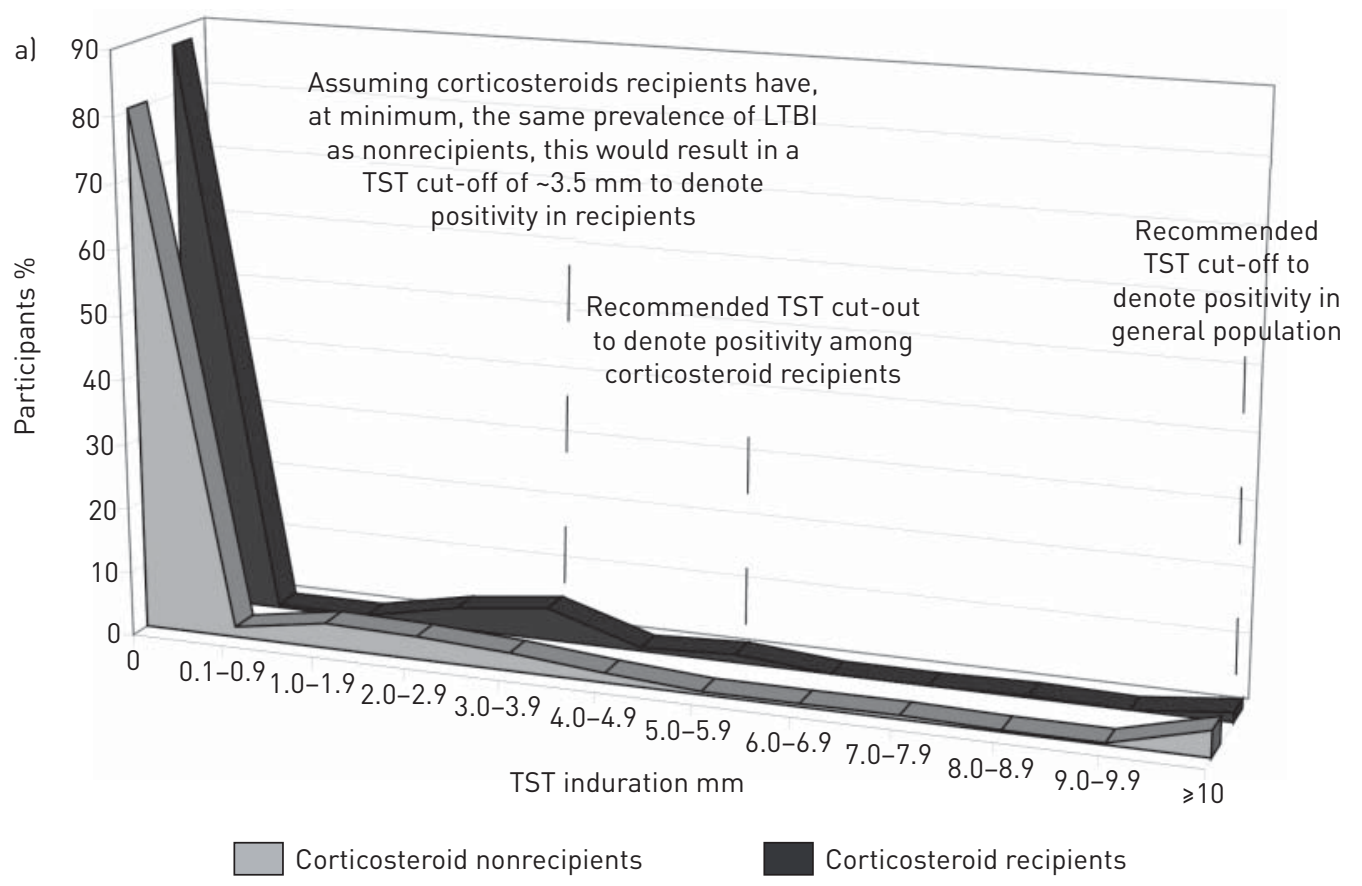

b)

\begin{tabular}{lccc}
\hline & $\begin{array}{c}\text { Corticosteroid\# } \\
\text { recipients }\end{array}$ & $\begin{array}{c}\text { Corticosteroid\# } \\
\text { nonrecipients }\end{array}$ & p-value \\
\hline Participants $\boldsymbol{n}(\%)$ & $93(1.3)$ & $7224(98.7)$ & \\
Prevalence of LTBI by NHANES TST & & & \\
$\geqslant 10 \mathrm{~mm}$ induration cut-off & $1 / 93(1.5)$ & $293 / 7224(4.1)$ & 0.21 \\
$\geqslant 5 \mathrm{~mm}$ induration cut-off+ & $3 / 93(3.1)$ & $443 / 7224(6.1)$ & 0.22 \\
$\begin{array}{l}\text { Prevalence of previous TST screening } \\
\text { Prevalence of chemoprophylaxis receipt for }\end{array}$ & $59 / 93(62.9)$ & $4936 / 6897(71.6)$ & 0.07 \\
$\begin{array}{l}\text { LTBI among individuals with a previously } \\
\text { reported positive TST }\end{array}$ & & & \\
$\begin{array}{l}\text { Individuals with } \geqslant 10 \mathrm{~mm} \text { induration on NHANES TST } \\
\text { Individuals with } \geqslant 5 \mathrm{~mm} \text { induration on NHANES TST }\end{array}$ & $0 / 0$ & $42 / 94(44.7)$ & 0.53 \\
\hline
\end{tabular}

FIGURE 1 a) Distribution of tuberculin skin test (TST) induration, and b) prevalence of latent tuberculosis infection (LTBI), screening and preventative therapy among corticosteroid recipients and nonrecipients. Data are presented as $\mathrm{n} / \mathrm{N}$ (\%) unless otherwise stated. NHANES: National Health and Nutrition Examination Survey. ": cortisone acetate, dexamethasone, fludrocortisone acetate, hydrocortisone, methylprednisolone, prednisolone, prednisolone sodium phosphate or prednisone; ${ }^{\circ}: \mathrm{n}=294(4.0 \%){ }^{+}: \mathrm{n}=446(6.1 \%)$.

vaccination in the corticosteroid group may, in part, explain the trends towards less screening and chemoprophylaxis in this group.

There are several limitations to the present study. First, it was based on data from 1999 to 2000 and more recent data were not available for analysis. Second, the small sample size of corticosteroid recipients may have impeded our ability to detect statistically significant differences with respect to TST screening and chemoprophylaxis receipt between the two groups. Increasing sample size numbers was not possible by us, as data collection was undertaken by the US Centers for Disease Control. Third, our purpose was not to determine if corticosteroid receipt was independently associated with LTBI diagnosis, screening and treatment, but instead to perform descriptive analysis, as oral corticosteroid receipt is already recognised by tuberculosis guidelines as a circumstance for targeted screening and treatment. The small sample size of corticosteroid recipients also precluded us from examining possible independent associations. Fourth, some individuals with a positive TST whom we considered to have LTBI may have been false positives due to 
previous BCG vaccination or nontuberculous mycobacterial infection. We elected not to exclude individuals with a history of BCG vaccination $(\sim 10 \%)$ from the analysis because we suspected that many individuals with BCG vaccination and a positive TST would still be true positives. We excluded individuals with any known or uncertain self-reported history of active tuberculosis to minimise TST positivity for this reason. We speculate that few, if any, participants would have had unknown active tuberculosis at the time of the survey TST. Fifth, although $83 \%$ of prescription medication data was confirmed by examiner inspection of prescription containers, there may have been some degree of misclassification between the corticosteroid recipient and nonrecipient groups in the remaining $17 \%$ of participants. Sixth, information on corticosteroid dose was not available. However, even individuals not taking a corticosteroid but having received one within the preceding 6 months have been found to have a significantly increased risk of developing active tuberculosis, if infected [3], and such individuals would have been classed as nonrecipients in our study. Information on drug duration was available in this study and about threequarters of recipients received corticosteroids for $\geqslant 2$ weeks, which is the guideline-reported minimum duration associated with increased active tuberculosis development. Seventh, information on previous active tuberculosis, TST screening and receipt of chemoprophylaxis for latent tuberculosis were all based on self-report, potentially introducing recall and social desirability biases. NHANES also collected no information relating to the reasons for nonscreening and nonreceipt of chemoprophylaxis (i.e. physicianversus patient-related factors). Finally, a newer, more specific and potentially more convenient method for one-time LTBI screening (i.e. serum interferon- $\gamma$ release assay) has emerged since this data collection.

In conclusion, the current guideline-recommended TST threshold of $\geqslant 5 \mathrm{~mm}$ to denote positivity among recipients of oral corticosteroids may need to be lowered to $\sim 3.5 \mathrm{~mm}$ in order to adequately capture LTBI in this at-risk group but further research with prospective data and adjustment for confounders is required. Fewer oral corticosteroid recipients were screened and treated for LTBI compared with nonrecipients, which may be placing this group at heightened risk. More frequent screening for LTBI and chemoprophylaxis of infected cases are probably needed among tuberculosis-vulnerable oral corticosteroid recipients. skin test cut-off http://ow.ly/xHZMZ

Nicholas T. Vozoris ${ }^{1,2}$, Julie Seemangal ${ }^{1}$ and Jane Batt ${ }^{1,2}$

${ }^{1}$ Tuberculosis Program, Division of Respirology, Dept of Medicine, St Michael's Hospital, Toronto, Canada. ${ }^{2}$ Dept of Medicine, University of Toronto, Toronto, Canada.

Correspondence: Nicholas T. Vozoris, Division of Respirology, Dept of Medicine, St Michael's Hospital, 30 Bond Street, Toronto, Ontario, M5B 1W8, Canada. E-mail: nick.vozoris@utoronto.ca

Received: March 292014 | Accepted after revision: June 032014 | First published online: June 252014

Conflict of interest: None declared.

\section{References}

1 American Thoracic Society, Centers for Disease Control and Prevention. Targeted tuberculin testing and treatment of latent tuberculosis infection. Am J Respir Crit Care Med 2000; 161: S221-S247.

2 Tuberculosis Prevention and Control, Public Health Agency of Canada, Canadian Lung Association/Canadian Thoracic Society. Canadian Tuberculosis Standards. 7th Edn. Ottawa, Minister of Health, 2013.

3 Jick SS, Lieberman ES, Rahman MU, et al. Glucocorticoid use, other associated factors, and the risk of tuberculosis. Arthritis Rheum 2006; 55: 19-26.

4 United States Department of Health and Human Services, Centers for Disease Control and Prevention, National Center for Health Statistics. NHANES 1999-2000 Public Data Release File Documentation. www.cdc.gov/nchs/ data/nhanes/nhanes_99_00/general_data_release_doc.pdf Date last accessed: February 17, 2014.

5 United States Department of Health and Human Services, Centers for Disease Control and Prevention, National Center for Health Statistics. National Health and Nutrition Examination Survey (NHANES), 1999-2000, NCHS User Guide - Questionnaire: Prescription Medications. www.icpsr.umich.edu/icpsrweb/ICPSR/series/39/studies/ 25501 Date last accessed: February 17, 2014.

6 Gabrielson AE, Good RA. Chemical suppression of adoptive immunity. Adv Immunol 1967; 6: 91-229.

7 Bovornkitti S, Kangsadal P, Sathirapat P, et al. Reversion and reconversion rate of tuberculin skin reactions in correction with the use of prednisone. Dis Chest 1960; 38: 51-55.

8 Schatz M, Patterson R, Kloner R, et al. The prevalence of tuberculosis and positive tuberculin skin tests in a steroidtreated asthmatic population. Ann Intern Med 1976; 84: 261-265.

9 Bélard E, Semb S, Ruhwald M, et al. Prednisolone treatment affects the performance of the QuantiFERON Gold In-Tube test and the tuberculin skin test in patients with autoimmune disorders screened for latent tuberculosis infection. Inflamm Bowel Dis 2011; 17: 2340-2349. 